\title{
Edge-balanced Index Sets of the Nested Graph with Power-cycle $C_{\mathrm{s}^{m}} \times P_{m_{\mathrm{s}}}$ (I)
}

\author{
Jinmeng Liu \\ Henan Polytechnic University, Jiaozuo, China \\ E-mail: xingfu0147@126.com \\ Yuge Zheng \\ Henan Polytechnic University, Jiaozuo, China \\ E-mail: zhengyuge@hpu.edu.cn
}

\begin{abstract}
Based on the power-cycle nested graph brought before, using the research methods and techniques of graph theory and combinatorial mathematics, through studying the new design idea about the basic graph,nested-cycle subgraph with gear and five-vertex sector subgraph-group, the edgebalanced index sets of the power-cycle nested graph $\boldsymbol{n}=5$ are provided here, for $m \equiv 1(\bmod 3)$ and $m \geq 4$, and the proofs of the computational of formulas and the construction of the corresponding graphs also give out.
\end{abstract}

Index Terms - Edge-friendly Labeling, Edge-balanced Index Set, Graph $C_{5^{m}} \times P_{m_{5}}$, The Nested-cycle Graph With Gear ,Five-vertices Sector Subgraph-group

\section{INTRODUCTION}

Graph theory is an important branch of discrete mathematics which regards figure as the research object. B.M.Stewart introduced a theory in 1966 which use the vertices of graph and the label function of edges making the vertices of graph correspond to the edges, through the mapping function and then studies the characteristics and inherent characteristics of all kinds of figure. Its theory can be applied to the information engineering, communication network, computer science, economics and management, medicine and so on. Over the years many domestic and foreign researchers devote themselves to this area, and have access to a series of results.

In 1995, M.C.Kong and others defined the edgebalanced graph and strong edge-balanced graph in reference [1], and put forward two conjectures: Conjecture 1: All trees except $S_{n}, n$ is odd, are edgebalanced. Conjecture 2: All connected regular graphs except $K_{2}$ are edge-balanced. The reference [2] extends the concept of edge-balanced labeling to multigraphs and completely characterizes the edge-balanced multigraphs, and proves the two conjectures are true in the reference [1], at the same time proves the problem of decide a graph is edge-balanced does not belong to NP-hard. In 2008, Alexander and Harris studied the vertex -balanced index and friendly index sets of the figure in the literature [3-5].

Since 2009, author and her student Juan Lu solved the edge-balanced index sets of chain graph. In 2010, Ying Wang started the research about the edge-balanced index sets of the equal-cycle nested graph [7].From 2010, Hongjuan Tian[8] and others began the study of edge-balanced index sets of the nested graph with power-cycle. With the increase of $n$, the difficulty of graph design of odd $n$ increases, ingenious design of classification is required for a nested transform edge index. In this paper, lay emphasis on the research about the graph when $n=5$.Using the innovative methods and techniques, categories design, proof method of fivevertices sector sub graph-group and other methods of Graph theory and Combinatorial mathematics, which have Completely solved the existence of the edgebalanced index set, construction methods and formulas proof. We choose different $\mathrm{n}$, the subgraph of construction is different in actual operation. The research of power-cycle nested graph $C_{5^{m}} \times P_{m_{5}}$ is divided into three parts.But in this paper we only research it when $m \equiv 1(\bmod 3)$ and $m \geq 4$.

The paper is organized as follows. In Section 2 ,we give the definitions that we shall consider. In section 3 includes the graphs with maximum edge-balanced index and using five-vertices sector subgraph-group to proof the lemmas. The main theorem is presented in Section 4. The final Section 5 contains the conclusion.

\section{PRELIMINARY NOTES}

In graph theory, a graph $G$ is an ordered pair $(V(G), E(G))$ consists of vertex set $V(G)$ and edge set $E(G)$, together with an incidence function that associates with each edge of $G$ an ordered pair of vertices of $G$.

Definition 1 In graph $G$, let: $f: E(G) \rightarrow Z$ be an edge labeling function, that is to say, $\forall e \in E(G)$ to define $f(e)=0$ or 1 .The edge set labeled 0 or 1 is recorded as $E(0)$ and $E(1)$, using $e(0), e(1)$ to present the number of $E(0), E(1)$. According to the edge 
labeling $f$, we can define $f^{+}: V(G) \rightarrow\{0,1\}$. If $e_{x}(0)$ is more than $e_{x}(1), f^{+}(x)=0$,the vertex $x$ is labeled 0 ;if $e_{x}(0)$ is less than $e_{x}(1), f^{+}(x)=1$, the vertex $x$ is labeled 1 ; otherwise, the vertex $x$ is unlabeled. $e_{x}(0)$ or $e_{x}(1)$ presents the radix number of the edge collection that the edges linked $x$ are labeled 0 or 1 .The radix number of the vertex set $V(0), V(1)$ is presented by $v(0)$ or $v(1)$.

Definition 2 Let: $f: E(G) \rightarrow Z$ is an edge labeling function of $G$. If $|e(0)-e(1)| \leq 1, f$ is considered as an edge-friendly labeling of the graph $G$.

Definition $3 \operatorname{EBI}(G)=\{|v(0)-v(1)|$ : the edge labeling $f$ is edge-friendly $\}$ is thought as the edgebalanced index set of the graph $G$, if there is an edgefriendly labeling $f$ in a graph $G$.

Definition $4 C_{5^{m}}$ shows the graph contains m cycles, and the number of the vertices is added by power exponent from the inner cycle to the outer.

Definition $5 P_{m_{5}}$ is a ray path that every road contains $\mathrm{m}$ points, and there are five branches at any points except the terminal point of each road.
Definition 6 The power-cycle nested graph $C_{5^{m}} \times P_{m_{5}}$ is the Cartesian product of $C_{5^{m}}$ and $P_{m_{5}}$.

Example. Fig. 1 illustrates two graphs of $C_{5^{m}} \times P_{m_{5}}$
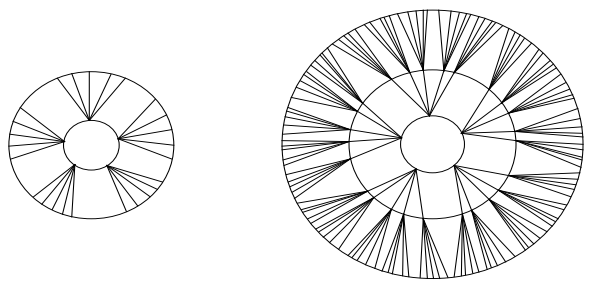

$$
C_{5^{2}} \times P_{2_{5}} \quad C_{5^{3}} \times P_{3_{5}}
$$

Fig. 1: two graphs of $C_{5^{m}} \times P_{m_{5}}$

For convenience, we will have the following label for the nested network graph: The vertices of the most inner circle in clockwise order are labeled as follows: $(1)_{1},(1)_{2},(1)_{3},(1)_{4},(1)_{5}$. Similarly, from inside to outside ,the vertices of the most outside circle in clockwise are labeled as follows: $(m)_{1},(m)_{2}, \cdots,(m)_{5^{m}-1},(m)_{5^{m}}$.

Among them, symbols $(j)_{i}$ says the $i$ th vertex in the $j$ th circle.The ray paths in the power-cycle nested graph, as follows:

$$
\begin{aligned}
& (1)_{i_{1}} \rightarrow(2)_{i_{2}} \rightarrow(3)_{i_{3}} \rightarrow \cdots \rightarrow(m-2)_{i_{m-2}} \rightarrow(m-1)_{i_{m-1}} \rightarrow(m)_{i_{m}} \\
& \quad\left(i_{k}=5\left(i_{k-1}-1\right)+j_{k-1} ; 1 \leq i_{1} \leq 5 ; 1 \leq j_{k-1} \leq 5 ; 2 \leq k \leq m\right) \\
& (1)_{1} \rightarrow(2)_{1} \rightarrow(3)_{1} \rightarrow \cdots \rightarrow(m-1)_{1} \rightarrow(m)_{1} ; \\
& (1)_{1} \rightarrow(2)_{1} \rightarrow(3)_{1} \rightarrow \cdots \rightarrow(m-1)_{1} \rightarrow(m)_{2} ; \\
& \quad \cdots \\
& (1)_{1} \rightarrow(2)_{5^{1}} \rightarrow(3)_{5^{2}} \rightarrow \cdots \rightarrow(m-1)_{5^{m-2}} \rightarrow(m)_{5\left(5^{m-2}-1\right)+4} ; \\
& (1)_{1} \rightarrow(2)_{5^{1}} \rightarrow(3)_{5^{2}} \rightarrow \cdots \rightarrow(m-1)_{5^{m-2}} \rightarrow(m)_{5^{m-1}} ; \\
& \quad \cdots \\
& (1)_{5} \rightarrow(2)_{5^{2}} \rightarrow(3)_{5^{3}} \rightarrow \cdots \rightarrow(m-1)_{5^{m-1}} \rightarrow(m)_{5\left(5^{m-1}-1\right)+4} ; \\
& (1)_{5} \rightarrow(2)_{5^{2}} \rightarrow(3)_{5^{3}} \rightarrow \cdots \rightarrow(m-1)_{5^{m-1}} \rightarrow(m)_{5^{m}}
\end{aligned}
$$

That is:

The above says that the $C_{5^{m}} \times P_{m_{5}}$ has $5^{m}$ paths.

Definition 7 A 1-vertex $x$ is considered to be saturated, if the $n$ edges linked to $x$ satisfy $e_{x}(1)=e_{x}(0)+1$, when $\mathrm{n}$ is an odd number; $e_{x}(1)=e_{x}(0)+2$, when $\mathrm{n}$ is an even number ;otherwise, the 1 -vertex $x$ is unsaturated.A 0 -vertex $x$ is considered to be saturated, if the $n$ edges linked to $x$ are all 0-edges; otherwise, the 0 -vertex $X$ is unsaturated.

Definition 8 Let $m=3 t+1\left(t \in N^{+}\right)$, the induced subgraph of the vertices, which the ray paths through the points with the vertices on the $3 t+1\left(t \in N^{+}\right)$cycle as starting points and the vertices on the $3(t+1)+1\left(t \in N^{+}\right)$.cycle as the terminal points,is thought as the nested-cycle subgraph,denoted by $V_{t}\left(t=1,2, \cdots, \frac{m-4}{3}\right)$. 
Definition 9 For the nested-cycle subgraph ,based on starting points of the ray paths, $V_{t}\left(t=1,2, \cdots, \frac{m-4}{3}\right)(m \geq 4)$ is subdivided into sector subgraph equally.If the ray path's starting points is on the $\partial$ th cycle.Then the nested-cycle subgraph can be divided into $5^{\partial}$ sector graphs,denoted separately as $S_{1}, S_{2}, \cdots, S_{5^{0}}$.

Definition 10 For the sector subgraphs in the given nested-cycle subgraph, denoted $S(i)=S_{5 i+1} \cup S_{5 i+2} \cup S_{5 i+3} \cup S_{5 i+4} \cup S_{5 i+5}\left(0 \leq i \leq 5^{\partial-1}-1\right)$ as fivevertices sector subgraph-group, and the label of the $S(i)$ is in the same, denoted by $S=S(i)$.

\section{LEMMAS AND PROOF}

Lemma 1 For the power-cycle nested graph $C_{5^{m}} \times P_{m_{5}}$, when $m=4, \max \left\{E B I\left(C_{5^{4}} \times P_{4_{5}}\right)\right\}=594$

Proof: Firstly, construct the maximum edge-balanced index about $C_{5^{4}} \times P_{4_{5}}$ :

Because the power-circle nested graph is nested by the power-circle graph and the ray paths graph. We will label respectively the power circle graph and the ray paths graph.

Step 1: We mark all edges as 0-edge which are in the paths of $\langle 1\rangle, 2\rangle,<3>$ and in the circles of $\langle 4\rangle,<5\rangle,<6>$.

$$
\begin{aligned}
& <1>(1)_{a}(2)_{b}(3)_{c}(4)_{d}\left(\begin{array}{l}
a \in[1,5] ; b=3+w+5 s, w \in[0,2], s \in[0,4] ; \\
c=11+w+25 s, w \in[0,14], s \in[0,4] ; \\
d=51+w+125 s, w \in[0,74], s \in[0,4]
\end{array}\right) \\
& <2>(2)_{b}(3)_{c}(4)_{d}\left(\begin{array}{l}
b=1+w+5 s, w \in[0,1], s \in[0,4] ; \\
c=5+w+25 s, w \in\{0,5\}, s \in[0,4] ; \\
d=21+w+125 s, w \in[0,4] \cup[24,29], s \in[0,4]
\end{array}\right) \\
& <3>(3)_{c}(4)_{d}\left(\begin{array}{l}
c=1+w+25 s, w \in[0,3] \cup[5,8], s \in[0,4] ; \\
d=1+5 w+125 s, w \in[0,3] \cup[5,8], s \in[0,4]
\end{array}\right)
\end{aligned}
$$

$<4>$ The 2th circle and the 3th circle.

$$
\begin{aligned}
& \langle 5\rangle(4)_{d}(4)_{d+1}(d=2+2 w+5 s+125 f, w \in[0,1] ; s \in[0,3] \cup[5,8] ; f \in[0,4]) \\
& \left\langle 6>(4)_{d}(4)_{d+1}(d=47+2 w, w \in[0,16])\right.
\end{aligned}
$$

Step 2: On the basis of step1,

(3) (4) $_{d}(c=11+w, w \in[0,5], d=51+w, w \in[0,29])$, $(2)_{2}(3)_{10},(3)_{10}(4)_{47},(3)_{10}(4)_{48},(3)_{10}(4)_{49}$ and
$(3)_{10}(4)_{50}$ have been marked 0-edges,then turn them from 0-edges to 1-edges.

Step 3: The other edges in the $C_{5^{4}} \times P_{4_{5}}$ are labeled as1.

In the graph, there are 1555 edges,of which 777 are the 0 -edges.According to the definition of edge-friendly labeling, $|e(0)-e(1)| \leq 1$, we can get the edge-friendly labeling of the labeled graph by calculation. All the vertices

$$
\begin{aligned}
& (2)_{i}(i=3+w+5 s, w \in[0,2], s \in[0,4]), \\
& (3)_{i}(i=5+25 w, w \in[0,4]),(3)_{i}(i=17+w, w \in[0,8]), \\
& \text { (3) }_{i}(i=35+w+25 s, w \in[0,15], s \in[0,3]) \text { are labeled as }
\end{aligned}
$$

0 . There are 930 -vertices,so $|v(1)-v(0)|=|687-93|=594$.

We can prove $E B I\left(C_{5^{4}} \times P_{4_{5}}\right)=594$ is the maximum of edge-balanced index in the graph $C_{5^{4}} \times P_{4_{5}}$.

In this labeled graph, the vertices on the first cycle have degree7.The vertices on the 4th cycle have degree 3 , and the others all have degree 8 . The edges linking the 0 -vertex are labeled 0 ; the 1 -vertex are all saturated, except that the vertex $(2)_{2}$, which links two 0 -edges.

In order to change the label of any 0 -vertex into 1vertex or unlabeled, we need to interchange 40 -edges and 1-edges at least, but any of the 1-vertex's label changes, we only need to interchange 20 -edges and 1edge at least. It is obvious that the interchanging must bring $v(0)$ increases or remains unchanged, and meanwhile $v(1)$ decreases definitely. Thus the value of $|v(1)-v(0)|$ reduces. That's to say:

$$
\max \left\{E B I\left(C_{5^{4}} \times P_{4_{5}}\right)\right\}=|v(1)-v(0)|=594
$$

Lemma 2 In the $C_{5^{m}} \times P_{m_{5}}$, for the five-vertices sector subgraph group $S, \max \{E B I(S)\}=589$.

Proof:Because these five-vertices sector subgraphgroup have the same characteristics of edge and vertex. We only give the label of $S=S(0)=S_{1} \cup S_{2} \cup S_{3} \cup S_{4} \cup S_{5}$, the other five-vertices $S(i)\left(1 \leq i \leq 5^{\partial-1}-1\right)$ sector subgraph-group have the same label as $S(0)$.

Firstly, construct the maximum edge-balanced index about $S$ :

Step 1: We mark all edges for 0-edge which are in the paths of $\langle 1>,<2>,<3>$ and in the circle of $<4>,<5>,<6>,<7>$.

$$
<1>(\partial)_{a}(\partial+1)_{b}(\partial+2)_{c}(\partial+3)_{d}\left(\begin{array}{l}
a \in[1,5] ; b=1+w+5 s, w \in[0,1], s \in[0,4] ; \\
c=1+w+25 s, w \in[0,9], s \in[0,4] ; \\
d=1+w+125 s, w \in[0,49], s \in[0,4],
\end{array}\right)
$$




$$
\begin{aligned}
& <2>(\partial+1)_{b}(\partial+2)_{c}(\partial+3)_{d}\left(\begin{array}{l}
b=3+w+5 s, w \in[0,2], s \in[0,4] ; \\
c=11+w+25 s, w \in[0,1] \cup[5,7] \cup[10,11], s \in[0,4] ; \\
d=51+w, w \in[0,9] \cup[25,39] \cup[50,59]
\end{array}\right) \\
& <3>(\partial+2)_{c}(\partial+3)_{d}\left(\begin{array}{l}
c=13+w+25 s, w \in[0,2] \cup[6,7] \cup[10,12], s \in[0,4] ; \\
d=61+w+125 s, w \in\{0,5,10,30,35,50,55,60\}, s \in[0,4]
\end{array}\right) \\
& <4>(\partial+2) t h . \\
& <5>(\partial+1)_{b}(\partial+1)_{b+1}\left(\begin{array}{l}
b=1+w+5 s, \\
w \in[0,1] \cup\{4\}, s \in[0,4]
\end{array}\right) \\
& <6>(\partial+3)_{d}(\partial+3)_{d+1}\left(\begin{array}{l}
d=62+2 w+5 s+125 f, w \in[0,1] \\
s \in[0,2] \cup[6,7] \cup[10,12], f \in[0,4]
\end{array}\right) \\
& <7>(\partial+3)_{d}(\partial+3)_{d+1}(d=1+2 w, w \in[0,4])
\end{aligned}
$$

Step 2: On the basis of step1,

$$
(\partial+2)_{c}(\partial+3)_{d}\left(\begin{array}{l}
c=1+w, w \in[0,1] \\
d=1+w, w \in[0,9]
\end{array}\right)
$$

have been marked 0-edges.We turn them to 1-edges.

Step 3:The other edges in the $S$ are labeled as 1 .

In the $S$ graph, there are 1500 edges, of which 775 are the 0 -edges.According to the definition of edge-friendly labeling, $|e(0)-e(1)| \leq 1$, we can get the edge-friendly labeling of the labeled graph by calculation. In this labeled graph, all the vertices

$$
\begin{aligned}
& (\partial+1)_{b}(b=1+w+5 s, w \in[0,1], s \in[0,4]) \\
& (\partial+2)_{c}(c=1+w+25 s, w \in[2,11] \cup[15,17] \cup[20,21], s \in[0,4]) \\
& (\partial+2)_{c}(c=26+w+25 s, w \in[0,1], s \in[0,3])
\end{aligned}
$$

are 0 -vertices, $v(0)=93$.In order to satisfy the index calculation of nested-cycle,the number of vertex of $S$ doses't include $(\partial)_{a}(a \in[1,5])$. Thus $|v(1)-v(0)|=|682-93|=589$.

We can prove $E B I(S)=589$ is the maximum of edge-balanced index in the graph $S$.

In this labeled graph, the vertices in $S$ are all saturated. In order to turn the label of any 0 -vertex to1-vertex or unlabeled, we need to interchange 40 -edges and 1edges at least, but any of the 1-vertex's label changes, only need to interchange 30 -edges and 1-edges at least.Obviously, if change 0 -vertex's label ,then $v(0)$ increases or remains unchanged, and meanwhile $v(1)$ decreases, so the value of $|v(1)-v(0)|$ reduces.

That's to say: $\max \{E B I(S)\}=589$.

Lemma 3 For the power-cycle nested graph $C_{5^{\mathrm{m}}} \times P_{m_{5}}$, when $m \equiv 1(\bmod 3)$ and $m \geq 4$,

$$
\max \left\{\operatorname{EBI}\left(C_{5^{m}} \times P_{m_{5}}\right)\right\}=\frac{19 \times 5^{m-1}+1}{4}
$$

Proof: For $m=4$, the formula can be proved by Lemma 1.

When $m>4$, firstly,construct the graphics with the maximum edge-balanced index of $C_{5^{m}} \times P_{m_{5}}$.

Obviously, $C_{5^{m}} \times P_{m_{5}}=C_{5^{4}} \times P_{4_{5}} \bigcup\left(\bigcup_{t=1}^{\frac{m-4}{3}} V_{t}\right)$, the maximum edge-balanced index labeling method of $C_{5^{4}} \times P_{4_{5}}$ is the same as we structure in lemma 1. For the nested-cycle subgraph $\bigcup_{i=1}^{\frac{m-4}{3}} V_{i}$ consists $\frac{5^{m-1}-125}{124} S$, the $S$ in the $V_{t}\left(t=1,2, \cdots, \frac{m-4}{3}\right)$, whose maximum edge-balanced index labeling method is the same as we construct in lemma 2.During the process of label, we know the $C_{5^{m}} \times P_{m_{5}}$ satisfies the friendly labeling, and the characteristic of vertices remain unchanged,so this graphics is the maximum edge-balanced index.

We shall prove $\max \left\{E B I\left(C_{5^{m}} \times P_{m_{5}}\right)\right\}=\frac{19 \times 5^{m-1}+1}{4}$.

According to lemma 2, we know $\max \{E B I(S)\}=589$, if the starting points at the $\partial t h=3 t+1\left(t \in N^{+}\right)$cycle,then the graph can be divided into $5^{\partial}$ sector subgraphs 
and $5^{\partial-1}$ five-vertices sector subgraph-group.That's to say $V_{t}\left(t=1,2, \cdots, \frac{m-4}{3}\right)$ has $5^{3 t}$ five-vertices sector
subgraph-group.By computing, we can get the maximum edge-balanced index of $C_{5^{m}} \times P_{m_{5}}$.

$$
\begin{aligned}
& \max \left\{E B I\left(C_{5^{m}} \times P_{m_{5}}\right)\right\}=\max \left\{E B I\left(C_{5^{4}} \times P_{4_{5}}\right)\right\}+\sum_{t=1}^{\frac{m-4}{3}} \max \left\{E B I\left(V_{t}\right)\right\} \\
& =594+589 \times 5^{3}+589 \times 5^{6}+\cdots+589 \times 5^{m-4} \\
& =\frac{19 \times 5^{m-1}+1}{4}
\end{aligned}
$$

The proof is completed.

Lemma 4 For $S$ in nested-cycle subgraph $V_{\frac{m-4}{3}}(m \geq 7),\{588,587, \cdots, 1,0\} \subset E B I(S)$.

Proof: In the lemma 2, we get $\max \{E B I(S)\}=589$.

We know all the five-vertices sector graph-group have the same label.Below transform is based on the biggest index labeled graph constructed from lemma 2, and the change of every step is based on the previous step .The $(2 k-1)_{r}(2 k)_{s} \leftrightarrow(2 k)_{s}(2 k)_{s+1}$ means that we exchange $(2 k-1)_{r}(2 k)_{s}$ and $(2 k)_{s}(2 k)_{s+1}$ which are 0 -edge and 1-edge respectively.

First, build an odd index set:

Step 1: In turn exchange

$$
(\partial+2)_{c}(\partial+3)_{d} \leftrightarrow(\partial+3)_{d}(\partial+3)_{d+1}\left(\begin{array}{l}
c \in[3,10], \\
d=11+w+5 s, \\
w \in[0,2], s \in[0,7]
\end{array}\right) \text {, we can }
$$

obtain 24 labeled graphs of odd edge-balanced indexes which are $\{587,585, \cdots, 543,541\}$.

Step 2: In turn exchange

$$
(\partial+3)_{c}(\partial+4)_{d} \leftrightarrow(\partial+3)_{d}(\partial+3)_{d+1}\left(\begin{array}{l}
c=26+w+25 s, \\
w \in[0,9], s \in[0,3] ; \\
d=126+w+5 s+125 f, \\
w \in[0,2], s \in[0,9], f \in[0,3]
\end{array}\right),
$$

we can obtain 120labeled graphs of odd edge-balanced indexes which are $\{539,537, \cdots, 299,301\}$.

Step 3: In turn exchange

$$
(\partial+3)_{c}(\partial+4)_{d} \leftrightarrow(\partial+3)_{d}(\partial+3)_{d+1}\left(\begin{array}{l}
c=11+w+25 s, \\
w \in[0,1] \cup[5,7] \cup[10,11], s \in[0,4] ; \\
d=51+w+5 s+125 f, \\
w \in[0,2], \\
s \in[0,1] \cup[5,7] \cup[10,11], f \in[0,4]
\end{array}\right),
$$

we can obtain 105 labeled graphs of odd edge-balanced indexes which are $\{299,333, \cdots, 93,91\}$.

Step 4: In turn exchange

$$
(\partial+2)_{c}(\partial+3)_{d} \leftrightarrow(\partial+3)_{d}(\partial+3)_{d+1}\left(\begin{array}{l}
c=13+w+5 s+25 f, \\
w \in[0,2], \quad s \in\{0,2\}, f \in[0,4] ; \\
d=61+5 w+125 s, \\
w \in[0,2] \cup[10,11], s \in[0,4]
\end{array}\right),
$$

we can obtain 25 labeled graphs of odd edge-balanced indexes which are $\{89,87, \cdots, 41\}$

Step5: In turn exchange

$$
(\partial+2)_{c}(\partial+2)_{c+1} \leftrightarrow(\partial+2)_{c+1}(\partial+3)_{d}\left(\begin{array}{l}
c=13+w+5 s+25 f, \\
w \in[0,1], \quad s \in\{0,2\}, f \in[0,4] ; \\
d=70+5 w+125 s, \\
w \in\{0,1,10,11\}, s \in[0,4]
\end{array}\right),
$$

we can obtain 20 labeled graphs of odd edge-balanced indexes which are $\{39,37, \cdots 3,1\}$

Even index set structures as follows:

Step1: Exchange $(\partial+3)_{1}(\partial+3)_{2} \leftrightarrow(\partial+2)_{1}(\partial+3)_{2}$, we can obtain one labeled graphs of even edge-balanced indexes which is $\{588\}$.

Step2:Repeat from the first to fifth step as we construct the odd index sets. Thus we can obtain these labeled graphs of even edge-balanced indexes which are respectively $\{586,584, \cdots, 2,0\}$.

The proof is completed.

Lemma 5 For every of $S$ in the nested-cycle subgraph,

$$
V_{t}\left(1 \leq t \leq \frac{m-7}{3}\right)(m \geq 10),\{588,587, \cdots, 1,0\} \subset E B I(S) .
$$

Proof: Below transform is based on the biggest index labeled graph constructed form lemma3, and every step all proceeds is based on the previous step to transform, which in turn constructs labeling graphs corresponding with the index.One of $S$ in the,is denoted as $S_{V_{t}}$.

We know the $(\partial)_{a}(a \in[1,5])$ of $S_{V_{t+1}}$ regards as the poinds on the $(\partial+3)$ th circle of $S_{V_{t}}$ when we 
calculate .One $S_{V_{t}}$ of $V_{t}$ corresponds to $125 S_{V_{t+1}}$, we select $117 S_{V_{t+1}}$ from $125 S_{V_{t+1}}$, we change them as the following order in turn exchange $(\partial+1)_{b+1}(\partial+1)_{b} \leftrightarrow(\partial+1)_{b}(\partial)_{a}\left(\begin{array}{l}a \in[1,5] \\ b=5+5 w, w \in[0,4]\end{array}\right)$, we can change 585 1-vertex on the third circle of $S_{V_{t}}$ into unlabeled, so that the index can decrease by 585, respectively 585 labeled graphs are obtained. By the lemma 2, $\max \{E B I(S)\}=589$,so $\{588,587, \cdots, 5\} \subset \operatorname{EBI}\left(S_{V_{t}}\right)$, then we select one $S_{V_{t}}$ again, in turn exchange

$$
(\partial+1)_{b+1}(\partial+1)_{b} \leftrightarrow(\partial+1)_{b}(\partial)_{a}\left(\begin{array}{l}
a \in[1,4] \\
b=5+5 w, w \in[0,3]
\end{array}\right)
$$

similarly, we can change 4 1-vertex on the third circle of $S_{V_{t}}$ into unlabeled,so that the index can decrease by 4,respectively 4 labeled graphs are obtained, so $\{4,321\} \subset \operatorname{EBI}\left(S_{V_{t}}\right)$. That is $\{588,587, \cdots, 0\} \subset \operatorname{EBI}\left(S_{V_{t}}\right)\left(1 \leq t \leq \frac{m-7}{3}\right)$.

The proof is completed.

Lemma 6 In nested-cycle subgraph $C_{5^{m}} \times P_{m_{5}}$, when $m=4,\{593,591, \ldots, 1,0\} \subset \operatorname{EBI}\left(C_{5^{4}} \times P_{4_{5}}\right)$.

Proof:Below transform is based on the biggest index labeled graph constructed form lemma 1 , and every step is based on the previous step to transform, which in turn constructs labeling graphs corresponding with the index.

First, build an even index set:

Step 1: In turn exchange

$$
(3)_{\mathrm{c}}(4)_{d} \leftrightarrow(4)_{d}(4)_{d+1}\left(\begin{array}{l}
c=1+w+5 s+25 f, \\
w \in[0,3], s \in[0,1], f \in[0,4] \\
d=1+5 w+25 s+125 f, \\
w \in[0,3], s \in[0,1], f \in[0,4]
\end{array}\right),
$$

we can obtain 40 labeled graphs of even edge-balanced indexes which are $\{592,590, \cdots, 516,514\}$.

Step 2: In turn exchange

$$
(3)_{\mathrm{c}}(4)_{d} \leftrightarrow(4)_{d}(4)_{d+1}\left(\begin{array}{l}
c=36+w+5 s, w \in[0,14], \\
s \in[0,3] ; \\
d=176+w+5 s+125 f, \\
w \in[0,2], s \in[0,14], f \in[0,3]
\end{array}\right),
$$

we can obtain 180 labeled graphs of even edgebalanced indexes which are $\{512,508, \cdots, 156,154\}$.

Step 3: In turn exchange

$$
(3)_{\mathrm{c}}(4)_{d} \leftrightarrow(4)_{d}(4)_{d+1}\left(\begin{array}{l}
c=30+5 w+25 s, \\
w \in[0,1], \quad s \in[0,3] ; \\
d=146+w+25 s+125 f, \\
w \in[0,2], s \in[0,1], f \in[0,3]
\end{array}\right),
$$

we can obtain 24 labeled graphs of even edge-balanced indexes which are $\{152,15,0, . ., 108106\}$.

Step 4: In turn exchange

$$
(3)_{c}(4)_{d} \leftrightarrow(4)_{d}(4)_{d+1}\left(\begin{array}{l}
c=17+w, w \in[0,8] ; \\
d=81+w+5 s, w \in[0,2], s \in[0,8]
\end{array}\right),
$$

we can obtain 27 labeled graphs of even edge-balanced indexes which are $\{104,102, \cdots, 54,52\}$.

Step5: In turn exchange

$$
(3)_{c}(3)_{c+1} \leftrightarrow(3)_{c}(4)_{d}\left(\begin{array}{l}
c=26+w+5 s+25 f, \\
w \in[0,2], \quad s \in[0,1], f \in[0,3] ; \\
d=126+5 w+125 s, \\
w \in[0,2] \cup[5,7], s \in[0,3]
\end{array}\right)
$$

we can obtain 24 labeled graphs of even edge-balanced indexes which are $\{50,48, \cdots, 6,4\}$.

Step6: In turn exchange

$$
(1)_{1}(2)_{1} \leftrightarrow(2)_{1}(2)_{2},(1)_{2}(2)_{6} \leftrightarrow(2)_{6}(2)_{7} \quad \text {,we }
$$
can obtain 2 labeled graphs of even edge-balanced indexes which are $\{2,0\}$.

Odd index set structures as follows:

Step1: Exchange $(1)_{1}(2)_{3} \leftrightarrow(1)_{1}(1)_{2}$,we can obtain one edge-balanced indexes which is $\{593\}$.

Step2: Repeat from the first to fifth step as we construct the odd index sets. Thus we can obtain these labeled graphs of even edge-balanced indexes which are respectively $\{586,584, \cdots, 2,0\}$.

Step3: Exchange $(1)_{1}(2)_{1} \leftrightarrow(2)_{1}(2)_{2}$,we can obtain one edge-balanced indexes which is $\{1\}$.

The proof is completed.

Lemma 7 In the nested graph with power-cycle $C_{5^{m}} \times P_{m_{5}}$, when $m \equiv 1(\bmod 3)$ and $m \geq 4$,

$$
\left\{\frac{19 \times 5^{m-1}-3}{4}, \frac{19 \times 5^{m-1}-7}{4}, \cdots, 1,0\right\} \subset \operatorname{EBI}\left(C_{5^{m}} \times P_{m_{5}}\right)
$$

Proof: (1)When $m=4$, the formula can be proved by lemma 6.

Now, we prove the formula was established when $m>4$. Below transform is based on the biggest index labeled graph constructed form lemma 3.

$$
\text { (2) When } m=7, C_{5^{7}} \times P_{7_{5}}=C_{5^{4}} \times P_{4_{5}} \cup V_{1} \text {, }
$$


Step 1: According to changing method of $S$ in lemma 4.By lemma 4,every $S_{v_{1}}$ 's index in $V_{1}$ can reduces by 589 , when we exchange, we can obtain 589 labeled graphs conversely, so $\{589,588, \cdots, 0\} \subset E B I(S)$.

$V_{1}$ has $125 S_{v_{1}}$, which have the same changing method as $S$ in lemma 4,so that the index can decrease by $589 \times 125$, when we exchange, we can obtain 73625 edge-balanced conversely.so $\{74218,74217, \cdots, 594\} \subset \operatorname{EBI}\left(C_{5^{5}} \times P_{7_{5}}\right)$

Step 2: To select $118 S_{v_{1}}$ from $V_{1}$, in turn exchange $(5)_{b+1}(5)_{b} \leftrightarrow(5)_{b}(4)_{a}\left(\begin{array}{l}a \in[1,5] \\ b=5+5 w, w \in[0,4]\end{array}\right)$, we can obtain 590 labeled graphs' edge-balanced indexes which are $\{593,592, \cdots, 4\}$, so $\{593,592, \cdots, 4\} \subset \operatorname{EBI}\left(C_{5^{7}} \times P_{7_{5}}\right)$

Step3: Then we select one $S_{v_{1}}$ from $V_{1}$, in turn exchange $(5)_{b+1}(5)_{b} \leftrightarrow(5)_{b}(4)_{a}\left(\begin{array}{l}a \in[1,4] \\ b=5+5 w, w \in[0,3]\end{array}\right)$, we can obtain 4 edge-balanced indexes which are $\{3,2,1,0\}$,so $\{3,2,1,0\} \subset E B I\left(C_{5^{7}} \times P_{7_{5}}\right)$.

(3) When $m \geq 10, C_{5^{\mathrm{m}}} \times P_{m_{5}}=C_{5^{4}} \times P_{4_{5}} \bigcup\left(\bigcup_{t=1}^{\frac{m-4}{3}} V_{t}\right)$,

Step 1: According to changing method of $S$ in lemma 4.By lemma 4,every $S_{\frac{V_{\frac{m-4}{3}}}{3}}$ 's index in $V_{\frac{m-4}{3}}$ can reduces by 589, when we exchange, we can obtain 589 labeled graphs conversely, $V_{\frac{m-4}{3}}$ has $5^{m-4} S_{V_{\frac{m-4}{3}}}$, which have the same change method as $S$ in lemma 4,so that the index can decrease by $589 \times 5^{m-4}$, when we exchange, we can obtain $589 \times 5^{m-4} \quad$ labeled graphs. So $\left\{\frac{19 \times 5^{m-1}-3}{4}, \frac{19 \times 5^{m-1}-7}{4}, \cdots, \frac{19 \times 5^{m-1}-2356 \times 5^{m-4}+1}{4}\right\} \subset \operatorname{EBI}\left(C_{5^{m}} \times P_{m_{5}}\right)$

Step 2: According to changing method of $S$ in lemma 5.All the $S$ of $V_{t}\left(1 \leq t \leq \frac{m-7}{3}\right)$ have the same changing method as $S$ in lemma 5,so that the index can decrease by $\frac{19 \times 5^{m-4}-2375}{4}$.That is

$$
\left\{\frac{19 \times 5^{m-4}-3}{4}, \frac{19 \times 5^{m-4}-7}{4}, \cdots, 595,594\right\} \subset E B I\left(C_{5^{m}} \times P_{m_{5}}\right) .
$$

Step 3: Repeat step 2 and step 3, when $m=7$, we can obtain 594 labeled edge-balanced graphs whose indexes are $\{593,592, \ldots, 0\}$, So

$$
\left\{\frac{19 \times 5^{m-1}-3}{4}, \frac{19 \times 5^{m-1}-7}{4}, \cdots, 1,0\right\} \subset E B I\left(C_{5^{m}} \times P_{m_{5}}\right)(m \geq 10) .
$$

The proof is completed.

\section{THEOREM}

According to lemma 3 and lemma7, we can get the following theorem.

Theorem 1: In the nested graph with power-cycle $C_{5^{m}} \times P_{m_{5}}$ when $m \equiv 1(\bmod 3)$ and $m \geq 4$,

$$
E B I\left(C_{5^{m}} \times P_{m_{5}}\right)=\left\{\frac{19 \times 5^{m}+1}{4}, \frac{19 \times 5^{m-1}-3}{4}, \cdots, 1,0\right\}
$$

\section{CONCLUSION}

In this paper ,in order to study the edge-balanced index sets of the nested graph with power-cycle $C_{5^{m}} \times P_{m_{5}}$,we introduced the five-vertices sector subgraph-group, showing the proof of the computational formula, at the same time giving the construction of the corresponding graphs. The resulting work using the same method will be investigated in a future paper.

\section{ACKNOWLEDGMENTS}

This work was financially supported by Applied Mathematics Provincial-level Key Discipline of Henan Province, Operational Research and Cybernetics Key Discipline of Henan Polytechnic University and the Education Department of Henan province science and technology research project.

\section{REFERENCES}

[1] Kong M, Sin-Min Lee. On Edge-Balanced Graphs[J],Graph Theory, Combinatoric and Algorithms, V.1,711 -722(1995).

[2] B.L.Chen, K.C. Huang and Shi-Shen Liu. On edge-balanced multigraphs, Journal of Combinatorial Mathematics and Combinatorial Computing,42 (2002),177-185.R. Nicole.

[3] AleLee and Ho Kuen Ng. On The Balance Index Set of Graphs[J]. Journal of Combina-torial Mathematics and Combinatorial Computing. 2008(66): 135-150.

[4] Harris Kwong, Sin-Min Lee and Ho Kuen Ng. On Friendly Index Sets of 2-Regular Graphs, Discrete Mathematics, 2008, 308: p. 5522-5532.

[5] Ebrahim Salehi and Sin-Min Lee, On Friendly Index Sets of Trees, Congressus Numerantium,2006, 178: p. 173-183.

[6] Juan Lu and Yuge Zheng: On the edge-balance index sets of $\mathrm{B}(\mathrm{n})$, Proceedings of the Jangjeon Mathematical Society, 12(1), 2009, 37-44.

[7] Ying Wang, Yuge Zheng and Sin-Min Lee: On the quick construction of all edge-balance index sets of $C_{n} \times P_{2} \quad(n \equiv 2,3(\bmod 4)) \quad[\mathrm{J}]$.Proceedings of the 
Jangjeon Mathematical Society.2010(13), No.3: 387-393.

[8] Yuge Zheng,Hongjuan Tian,On the Edge-Balance Index Sets of the Power Circle Nested Graph C2m $\times \mathrm{Pm} 2(\mathrm{~m} \equiv 0(\bmod 2))$, Advanced Science Letters, Volume 72012 , pp. 534-536(3).

\section{Authors' Profiles}

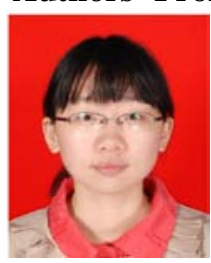

Jinmeng Liu: Post-graduated student for master degree for Applied Mathematics in Henan Polytechnic University, major in Graph theory and Combinatorial mathematics.

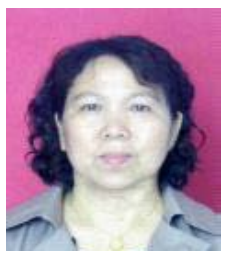

Yuge Zheng: Professor of Henan Polytechnic University, interested in Graph theory and Combinatorial mathematics. 